\title{
To robot or not to robot: Is that really the question?
}

A well-performed pulmonary lobectomy is the most effective way to treat primary non-small cell lung cancer. But, not all lobectomies are equal. Lobectomy can be performed via thoracotomy, sternotomy, or video or robotic assistance. Institutions differ in the approach to patient selection, preoperative preparation, anesthetic and technical surgical management, pathologic assessment, and postoperative care and surveillance.

Historic trends seem to support that minimally invasive lobectomy has advantages over lobectomy performed through thoracotomy. Pain and debility are less. Blood loss and length of stay may be reduced. Operative times and oncologic outcomes are equivalent. Video-assisted thoracoscopic surgery (VATS) appears to be less expensive than open surgery; robotics is most expensive.

Robotic advocates claim surgical robotics is better than VATS. Historically, robotic surgery provided 3-dimensional and high-definition visibility. Now VATS features both. Robotics also provides multiple angles of instrument rotation that allows numerous approaches for a given problem. This means that more complex procedures can be performed safely in small spaces.

So, does the use of robotic technology to perform lobectomy translate into superior outcomes?

It is not clear.

In the article by Dr Cerfolio and colleagues, ${ }^{1}$ the prospective robotic lobectomy experience of 4 surgeons at different institutions is reported to have a low operative mortality and morbidity with a length of stay half of VATS and a long-term survival that appears, at least, to be as good as open thoracotomy. ${ }^{1}$ By collectively reporting results, there is a larger number of patients that might clarify the role of robotics in the performance of lobectomy for lung cancer.

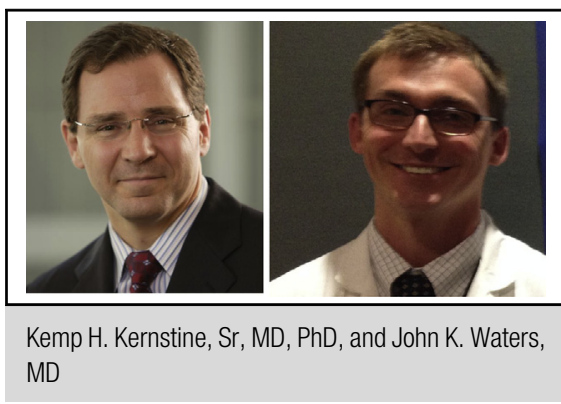

Central Message

Computer-assisted surgical technologies will continue to evolve and have a greater role in surgical management.

See Article page 778 .

Unfortunately, there is bias in the analysis. First is the choice of the 4 surgeons. It would have been preferable to randomly select the robotic surgeons. Furthermore, each surgeon and institution has different biases that are applied at multiple areas of patient management and long-term surveillance. Without agreed upon definitions and standardized ways of evaluating and managing patients, it is difficult to discern the role of robotic surgery versus the role of each institution's excellence in managing these patients.

If greater numbers are needed to better understand the role of robotics, the article by Kent and colleagues ${ }^{2}$ has addressed that concern in an unbiased fashion. But again, it does not provide a full understanding of the impact of using a surgical robot to perform lung cancer resections. What does seem to stand out is the fact that survival from experienced robotic lobectomy surgeons appears to be consistently good.

So, the robot can provide a safe minimally invasive surgical option. What's next? We should expect continued superior visibility with improvements in the capability of the robotic videoscope. The videoscope and instrument size should continue to decrease with retained ability to perform fine detailed work with greater arcs of motion. Ideally, a single-robotic port entry system is forthcoming.

Cerfolio and colleagues ${ }^{1}$ have shown that robotic lobectomy can be performed safely and effectively. Perhaps the greatest takeaway from this is that computer-assisted technology is maturing and will likely become an even greater part of our surgical armamentarium. 


\section{References}

1. Cerfolio RJ, Ghanim AF, Dylewski M, Veronesi G, Spaggiari L, Park BJ. The long-term survival of robotic lobectomy for non-small cell lung cancer: a multi-institutional study. J Thorac Cardiovasc Surg. 2018;155:778-86.
2. Kent M, Wang T, Whyte R, Curran T, Flores R, Gangadharan S. Open, videoassisted thoracic surgery, and robotic lobectomy: review of a national database. Ann Thorac Surg. 2014;97:236-44. 